\title{
Nonlinear saturation of Rayleigh-Taylor instability and generation of shear flow in equatorial spread-F plasma
}

\author{
N. Chakrabarti ${ }^{1,2}$ and G. S. Lakhina ${ }^{1}$ \\ ${ }^{1}$ Indian Institute of Geomagnetism, Dr. Nanabhai Moos marg, Colaba, Mumbai 400 005, India \\ ${ }^{2}$ Saha Institute of Nuclear Physics, Plasma Physics Division, Bidhannagar, Calcutta 700 064, India
}

Received: 20 June 2000 - Accepted: 20 September 2000

\begin{abstract}
An analysis of low order mode coupling equations is used to describe the nonlinear behavior of the RayleighTaylor (RT) instability in the equatorial ionosphere. The nonlinear evolution of RT instability leads to the development of shear flow. It is found that there is an interplay between the nonlinearity and the shear flow which compete with each other and saturate the RT mode, both in the collisionless and collisional regime. However, the nonlinearly saturated state, normally known as vortices or bubbles, may not be stable. Under certain condition these bubbles are shown to be unstable to short scale secondary instabilities that are driven by the large gradients which develop within these structures. Some understanding of the role of collisional nonlinearity in the shear flow generations is also discussed.
\end{abstract}

\section{Introduction}

The Rayleigh-Taylor (RT) instability has been extensively studied in a wide range of physical contexts both experimentally and theoretically. In spite of a long history of investigations there are many important motivations which still attract attention to different branches of physics, namely astrophysics (Arons et al., 1976), plasma fusion (Finn, 1993), space (Amatucci et al., 1996; Penano et al., 1998), atmospheric (Sazonov, 1991) and geophysics (Wilcock and Whitehead, 1991), etc. The primary source by which this instability is triggered is the gravitational force acting on an inverted density gradient (e.g. a heavy fluid supported by a light fluid). The basic mechanism of this instability, an interchange of flux tube to tap the gravitational free energy, is the same mechanism that drives the Rayleigh-Benard instability in the thermal convection of a gravitationally unstable fluid. In this case the mean temperature gradient of the fluid plays a similar role as the density gradient and the buoyancy force acts similar to the gravity. Apart from fluid dynamics RT

Correspondence to: G. S. Lakhina

(lakhina@iig.iigm.res.in) mode exists in magnetized plasmas in both collisional and collisionless regimes. For example, in laboratory plasma, RT mode arises due to an unfavorable curvature in the magnetic field in the presence of a pressure gradient. Sometimes this is known as curvature driven interchange mode (Pogutse et al., 1994). The RT instability is believed to cause the intense nighttime equatorial $\mathrm{F}$ region turbulence, known as equatorial spread F (Basu, 1997). Thus, we expect that this work has a much wider application than might be sought from specific equations under consideration. A considerable amount of work has been done in this area, and yet there is an incompatibility of theoretical and experimental results which suggests the possibility of obtaining more physics via nonlinear analysis and computer simulations.

As we will see due to the presence of the nonlinearities, the RT mode dynamics becomes more complicated. A complete theory of the full nonlinear system is very difficult. However, a self consistent numerical simulation might improve our understanding. Still the question remains what can be done analytically? To help us answer this question, we do have some methods for treating certain aspects of complex nonlinear behavior of a plasma. One such method, the low order mode coupling effect, 'Galerkin approximation', is used in this study. The present day strategy for a better theoretical understanding is to apply different levels of perception and knowledge. A 'coarse grained' view will lead to a general feeling of which processes are important. More detailed and narrow views on specific processes are necessary for the understanding of the 'elementary processes'. They will thereby lead to an estimate of the potential that the various 'elementary processes' do have in the overall dynamical evolution. In this work, we concentrate on 'simple models' which have proven to be good candidates for modeling some of the 'elementary processes'. However, we should not expect that mathematical rigorosity can go along with broad quantitative predictions for experiments/observations. The main aim is to sharpen our understanding of the principal processes that are possible due to nonlinearities. It will be emphasized that the 'simple models' are quite rich in their phenomenological 
aspect. Due to their low-dimensional nature, our increasing knowledge of nonlinear dynamics allows us to analyze their behavior in detail. Many aspects, which previously were thought to follow only from very complicated descriptions, can be explained by simple models.

Rayleigh-Taylor instabilities, as they are applied to the ionosphere, can be divided into two categories: collisional and inertial. In the collisional limit, the ion-neutral frequency is dominant, and $v_{i n} \gg \omega$, where $v_{i n}$ is the ion-neutral collision frequency and $\omega$ is the mode frequency; in the inertial limit, $\omega \gg v_{i n}$ and neutral collision may be neglected. Most of the research for nonlinear RT mode in the ionosphere has been restricted to collisional domain, although it has been shown that inertial effects are important in the high-latitude ionosphere. The influence of transverse velocity shear on Rayleigh-Taylor instability has been well investigated in the linear theory by various authors, especially, Guzdar, Satyanarayana, and their collaborators (Guzdar et al., 1982; Satyanarayana et al., 1987). They have found that a sheared velocity flow can substantially reduce the growth rate of a Rayleigh-Taylor instability in the short wavelength regime. They have also discussed the application of this result in ionospheric plasma (e.g. equatorial spread $\mathrm{F}$ and ionospheric plasma clouds). It is also known that RT mode may self consistently generate a velocity shear which can then stabilize the mode (Finn, 1993). In this report we study the effect of self generated velocity shear on the Rayleigh-Taylor mode to gain a better understanding of the theories of turbulence and transport studies.

In the literature there exists a systematic numerical simulation (Zalesak et al., 1982) for the evolution of the spread $\mathrm{F}$ bubbles consistent with experimentally measured ESF environment. This phenomena is explained in terms of the nonlinear evolution of the gravitationally driven collisional RT instability. Results indicate that there is a possibility of a secondary instability in the presence of an eastward neutral wind. However, in our analysis, we have pointed out that the large-scale bubble development of strong gradients could be the source of a 'secondary instability'. Therefore, following the similar argument given in Burlaga (1991), we can suggest that the formation and destruction of coherent structures in a turbulent state of ESF is indicative of intermittency. Motivated by these facts, we have carried out an analytical study of equilibrium and stability of nonlinearly generated RT bubbles in this paper. We have taken the point of view that the transition to turbulence may be due to the formation of coherent vortices by nonlinear saturation effects and their subsequent destruction due to excitation of fine scale secondary instabilities when certain critical conditions are met. The coherent nonlinear vortex solutions of the Rayleigh-Taylor wave have been investigated by several authors in one and two dimensions. Linear and nonlinear studies on the RT instability have been carried out in recent years by Flaherty, Finn, and others (Flaherty et al., 1999; Finn et al., 1992). In the past, it has been shown by many authors (Guzdar et al., 1982; Satyanarayana et al., 1987) that RT instability of a magnetized plasma may be saturated by the external impo- sition of velocity shear. Recently, in a complementary investigation, Finn (1993) has demonstrated by numerical simulations that the velocity shear may be self consistently generated since the RT vortices are themselves unstable to formation of velocity shear. The observed process of shear flow generation is the combination of several processes, but the most 'robust' processes are density profile flattening via nonlinear convection, and the generation of a shear component via Reynolds stresses. According to the simulation results, these processes seem to be irrelevant to the particular details of the plasma density and potential structures. Therefore, their description in terms of a low-dimensional model, which we will be considering here, is quite reasonable. As it has already been mentioned, such a model could help in scanning a wider range of plasma parameters and elucidate the physics of the phenomenon.

The rest of the paper is organized as follows. In Sect. 2, a simple physical model for RT instability is presented and the basic equations are derived. In Sect. 3, we have rederived the dispersion relation for the RT mode and its collisionless and collisional branch. In Sect. 4, a few mode representation is outlined and the nonlinear evolution of collisionless and collisional RT mode is presented analytically. In Sect. 5, saturation effect of velocity-shear is shown more realistically. In Sect. 6, a model calculation is presented for the demonstration of secondary instability due to bubbles. The role of collisional nonlinearity in the flow generation is discussed in Sect. 7. We conclude in Sect. 7 with a discussion of our results.

\section{Model and basic equations}

Development of a total self consistent and comprehensive theory which describes the nonlinear properties of RT mode applied to the ionosphere is very difficult in many aspects. In this report we have taken the vastly used and best illustrated model existing in the literature (Hassam et al., 1986; Keskinen et al., 1979).

The nonlinear fluid equations used to describe the electrostatic RT instability are the continuity and momentum equations for the electrons and ions. We model these equations in a three-dimensional slab geometry with the ambient magnetic field directed in the $z$ direction. For the purpose of relating this geometry to the ionospheric situations we employ the standard convention where $x$ is vertically upward (in the direction of the ambient density gradient), $N_{0}=N_{0}(x)$, $N_{0}^{-1} d N_{0} / d x=L_{n}^{-1}>0$, where $L_{n}$ is the density gradient scale length and $y$ is eastward which will be referred to as the horizontal or zonal direction. The gravitational acceleration is in the $-x$ direction $\left(g=-g \boldsymbol{e}_{x}\right)$. For this situation the plasma is unstable to RT instability, since $\boldsymbol{g} \cdot \nabla N_{0}<0$.

For the system described above, the flute type $(\partial / \partial z=0$, no variation along the magnetic field), RT instability is expected to be the fastest growing instability. The fluid equations can be simplified by the following assumptions:

1. We assume, that electrons are warm and ions are cold. 
The assumption of cold ions, i.e. the neglect of the ion temperature, is a shortcoming, because this eliminates the ion diamagnetic drift which introduces the finite Larmor radius effect (FLR) that could play a stabilizing role on RT instability, particularly in the short wavelength regime $k \rho_{i} \gg 1$, (where $\rho_{i}$ is the mean ion Larmor radius and $k$ is a typical wave number) (Huba et al., 1996). One advantage of setting $T_{i} \ll T_{e}$ is that the kinetic effect, such as Landau damping, will play no role and the system is well describe by the fluid model.

2. The wave electric field is virtually electrostatic, since the plasma pressure is much smaller than ambient magnetic energy density;

3. The electron and ion densities are equal (quasi-neutrality condition), since we are interested in the wavelengths much larger than Debye length;

4. We assume that a background neutral density exists with $v_{i n}$, the ion-neutral collision frequency and the electronneutral collision frequency may be neglected. Since in the $\mathrm{F}$ region $v_{e n} / \Omega_{e} \ll v_{i n} / \Omega_{i}\left(\Omega_{j}\right.$ is the cyclotron frequency of the species $j$ ), we assume $v_{i n} \ll \Omega_{i}$;

5. The waves are assumed to propagate exactly perpendicular to the ambient magnetic field, since these waves suffer the least diffusive damping;

6. The electron inertia is neglected, whereas the ion inertia is retained. It is shown that finite ion inertia polarization drift plays a significant role in the evolution of the RT instability in the inertial regime $\omega>v_{i n}$, where $\omega$ is the mode frequency.

With these restrictions, the RT instability in the F region of the ionosphere can be described by the following set of equations (Huba et al., 1986):

$$
\begin{aligned}
& \frac{\partial n}{\partial t}+\nabla \cdot\left(n \boldsymbol{V}_{j}\right)=0, \\
& 0=-\frac{e}{m_{e}}\left(\boldsymbol{E}+\frac{1}{c} \boldsymbol{V}_{e} \times \boldsymbol{B}\right)-\frac{T_{e}}{m_{e}} \frac{\nabla n}{n} \\
& \left(\frac{d}{d t}+v_{i n}\right) \boldsymbol{V}_{i}=\frac{e}{m_{i}}\left(\boldsymbol{E}+\frac{1}{c} \boldsymbol{V}_{i} \times \boldsymbol{B}\right)+\boldsymbol{g} \\
& \nabla \cdot \boldsymbol{J} \equiv \nabla \cdot\left[n e\left(\boldsymbol{V}_{i}-\boldsymbol{V}_{e}\right)\right]=0,
\end{aligned}
$$

where the various symbols have their usual meaning. Solving electron momentum Eq. (2) for their directed velocities, we find, to the lowest order

$$
\begin{aligned}
\boldsymbol{V}_{e}=\boldsymbol{V}_{e 0} & +\tilde{\boldsymbol{V}}_{e}=-\frac{c T_{e}}{e B L_{n}} \boldsymbol{e}_{y}+\frac{c}{B} \boldsymbol{e}_{z} \times \nabla \tilde{\phi} \\
- & \frac{c T_{e}}{e B} \boldsymbol{e}_{z} \times \nabla\left(\frac{\tilde{n}}{n_{0}}\right)-\frac{\nu_{e i}}{\Omega_{e}} \frac{c_{s}^{2}}{\Omega_{i}} \nabla\left(\frac{\tilde{n}}{n_{0}}\right),
\end{aligned}
$$

which consists of equilibrium diamagnetic drift and perturb $\boldsymbol{E} \times \boldsymbol{B}$ and the diamagnetic drift velocity. The last term arises due to the frictional force between electrons and ions; $T_{e}$ is the electron temperature and $\phi(\boldsymbol{E}=-\nabla \phi)$ is the electrostatic potential. Similarly, the ion velocity is determined from Eq. (3) and is given by

$$
\begin{aligned}
\boldsymbol{V}_{i}=\boldsymbol{V}_{i 0} & +\tilde{\boldsymbol{V}}_{i}=\frac{g}{\Omega_{i}} \boldsymbol{e}_{y}+\frac{c}{B} \boldsymbol{e}_{z} \times \nabla \tilde{\phi} \\
& -\left(\frac{d}{d t}+v_{i n}\right) \nabla_{\perp} \tilde{\phi}-\frac{v_{e i}}{\Omega_{e}} \frac{c_{s}^{2}}{\Omega_{i}} \nabla\left(\frac{\tilde{n}}{n_{0}}\right),
\end{aligned}
$$

where the terms on the right-hand side are equilibrium gravitational drift and perturb $\boldsymbol{E} \times \boldsymbol{B}$ drift, polarization drift, Pedersen drift and drift due to electron and ion friction, respectively and $d / d t \equiv \partial / \partial t+\boldsymbol{V}_{i} \cdot \nabla$. In solving $\tilde{\boldsymbol{V}}_{i}$, we have neglected ion viscous term $\left(\sim v_{i i}\right)$, since they are smaller than Pedersen drift contribution $\left(\sim v_{i n}\right)$. Here $c_{s}^{2}=T_{e} / m_{i}$ is the ion sound speed. By substitution of these velocities into Eqs. (4) and (1), we arrive at the coupled set of equations for the normalized potential $(\phi)$ and density $(n)$ fluctuations:

$$
\begin{aligned}
& \left(\frac{\partial}{\partial t}+\boldsymbol{e}_{z} \times \nabla \phi \cdot \nabla\right) \nabla_{\perp}^{2} \phi-\frac{\partial n}{\partial y}+v_{i n} \nabla n \cdot \nabla \phi= \\
& -v_{i n} \nabla_{\perp}^{2} \phi, \\
& \left(\frac{\partial}{\partial t}+\boldsymbol{e}_{z} \times \nabla \phi \cdot \nabla\right) n-\frac{\partial \phi}{\partial y}=D \nabla^{2} n .
\end{aligned}
$$

Note that in the density equation (8), $D=v_{e i} \rho_{e}^{2}$ arises, due to electron ion collisions that introduce an effective damping rate. Where $\rho_{e}=v_{t e} / \Omega_{e}, v_{t e}=\sqrt{T_{e} / m_{e}}$ and $\Omega_{e}=$ $\left(e B / m_{e} c\right)$. It may be seen that Eqs. (7) and (8) are same as the basic equations in Hassam et al. (1986), except for the $\nabla n \cdot \nabla \phi$ term in (7). The variables transform as follows:

$$
\begin{aligned}
& t \sqrt{\frac{g}{L_{n}}} \rightarrow t ; \quad \frac{x, y}{L_{n}} \rightarrow x, y ; \frac{\tilde{n}}{n_{0}} \rightarrow n ; \frac{c}{B} \sqrt{\frac{L_{n}}{g}} \frac{\tilde{\phi}}{L_{n}^{2}} \rightarrow \phi \\
& v_{i n} \sqrt{\frac{L_{n}}{g}} \rightarrow v_{i n} ; \frac{D}{L_{n}^{2}} \sqrt{\frac{L_{n}}{g}} \rightarrow D .
\end{aligned}
$$

\section{Linear instability}

Here we shall recapitulate the local $\left(k_{x} L_{n} \gg 1\right)$ linear dispersion of the RT mode that we are interested in via two limiting cases: inertial $\left(\omega \gg v_{i n}\right)$ and collisional $\left(v_{i n} \gg \omega\right)$ (Sudan and Keskinen, 1984). The linear dispersion equation of the system is obtained by linearizing Eqs. (7) and (8) and assuming that the perturbed quantities vary as $\exp \left(i k_{x} x+i k_{y} y-\right.$ $i \omega t$ ), where $k_{x}$ and $k_{y}$ are positive integers. The dispersion equation is given by

$$
\omega^{2}+i\left(v_{i n}+D k_{\perp}^{2}\right) \omega+\left(\frac{k_{y}^{2}}{k_{\perp}^{2}}-k_{\perp}^{2} D v_{i n}\right)=0,
$$

where $k_{\perp}^{2}=k_{x}^{2}+k_{y}^{2}$. The boundary between linearly stable and unstable regions of $\boldsymbol{k}$ space is given by $\nu_{i n} D=k_{y}^{2} / k_{\perp}^{4}$. The maximum growth rate is obtained for $k_{x}=0$. 
In the absence of any dissipation in the inertial regime, we find the dispersion relation

$\omega^{2}+\frac{k_{y}^{2}}{k_{\perp}^{2}}=0$

which gives the growth rate in the inertial regime as $\gamma_{i}=\left(k_{y} / k_{\perp}\right)\left(g / L_{n}\right)^{1 / 2}$. For $D=0$, and a finite ionneutral collision effect, the dispersion relation may be written as $\omega^{2}+i v_{i n} \omega+k_{y}^{2} / k_{\perp}^{2}=0$ from which we find

$\omega=-\frac{i v_{i n}}{2} \pm \frac{i v_{i n}}{2}\left[1+\frac{4 k_{y}^{2}}{k_{\perp}^{2} v_{i n}^{2}}\right]$.

For an ion-neutral collision dominated regime $v_{i n} \gg 1$ taking a positive sign, we can find that the collisional growth rate of the RT mode is $\gamma_{c}=\left(k_{y} / k_{\perp}\right)^{2}\left(g / L_{n} v_{i n}\right)$.

In the next section we would like to study the nonlinear evolution of these instabilities using truncated Fourier mode representation.

\section{Few-mode representation}

For RT turbulence we represent the state of the system with two complete fluctuation dynamics $\left(\phi_{1}, n_{1}\right)$ and $\left(\phi_{2}, n_{2}\right)$ with $\boldsymbol{k}_{\mathbf{1}}=\left(k_{x}, k_{y}\right)$ and $\boldsymbol{k}_{\mathbf{2}}=\left(2 k_{x}, k_{y}\right)$. The consideration of mode coupling terms then shows that the convective nonlinearity of the vorticity and the density drives the convective flows $\phi_{0} \sin \left(k_{x} x\right)$ and flattening of the background density gradient $n_{0} \sin \left(2 k_{x} x\right)$. All higher order components $\left(2 k_{x}, 2 k_{y}\right),\left(3 k_{x}, 0\right)$, etc. are truncated (Galerkin approximation). It is also assumed that the high $k$ modes are heavily damped by normal dissipation, such as viscosity and/or diffusion. The procedure is originally proposed by Howard and Krishnamurty in fluid dynamics literature (Howard and Krishanamurti, 1986) and later used by many others. The potential and density are represented by

$$
\begin{aligned}
& \phi=\phi_{0} \sin \left(k_{x} x\right)+\phi_{1} \sin \left(k_{x} x\right) \sin \left(k_{y} y\right) \\
&+\phi_{2} \sin \left(2 k_{x}\right) \cos \left(k_{y} y\right), \\
& n=n_{0} \sin \left(2 k_{x} x\right)+n_{1} \sin \left(k_{x} x\right) \cos \left(k_{y} y\right) \\
&+n_{2} \sin \left(2 k_{x} x\right) \sin \left(k_{y} y\right) .
\end{aligned}
$$

We would like to emphasize that the $\phi_{0}$ and $n_{0}$ terms in Eqs. (10) and (11) represent the driven convected (sheared) flow and flattening of density, respectively, arising essentially from the nonlinear interactions of the different modes (see Eqs. 12 and 15). Therefore, $\phi_{0}$ and $n_{0}$ should not be confused with the equilibrium value of the potential, $\phi$, and number density, $n$. Substituting Eqs. (10) and (11) in the basic equations (7) and (8) leads to the following dynamical evolution equations for the Fourier coefficients:

$$
\begin{aligned}
\frac{d \phi_{0}}{d t} & =-\frac{3}{4} k_{x} k_{y} \phi_{1} \phi_{2}-v_{i n} \phi_{0}, \\
\frac{d \phi_{1}}{d t} & =\frac{k_{y}}{k_{1}^{2}} n_{1}+\frac{k_{x} k_{y}\left(3 k_{x}^{2}+k_{y}^{2}\right)}{2 k_{1}^{2}} \phi_{0} \phi_{2}-v_{i n} \phi_{1},
\end{aligned}
$$

$$
\begin{aligned}
\frac{d \phi_{2}}{d t} & =-\frac{k_{y}}{k_{2}^{2}} n_{2}-\frac{k_{x} k_{y}^{3}}{2 k_{2}^{2}} \phi_{0} \phi_{1}-v_{i n} \phi_{2}, \\
\frac{d n_{0}}{d t} & =\frac{1}{2} k_{x} k_{y} n_{1} \phi_{1}-4 k_{x}^{2} D n_{0}, \\
\frac{d n_{1}}{d t} & =k_{y} \phi_{1}-k_{x} k_{y}\left(n_{0} \phi_{1}+\frac{1}{2} n_{2} \phi_{0}\right)-k_{1}^{2} D n_{1}, \\
\frac{d n_{2}}{d t} & =-k_{y} \phi_{2}+\frac{1}{2} k_{x} k_{y} n_{1} \phi_{0}-k_{2}^{2} D n_{2},
\end{aligned}
$$

where $k_{1}^{2}=k_{x}^{2}+k_{y}^{2}$ and $k_{2}^{2}=\left(2 k_{x}^{2}+k_{y}^{2}\right)$.

The above six ordinary differential equations have many nice properties which we will discuss in this paper. Before going to the analysis, we rewrite the Eqs. (12)-(17) in a simplified scaled form:

$$
\begin{aligned}
& \left(\frac{d}{d t}+v_{i n}\right) \widehat{\phi}_{0}=-\widehat{\phi}_{1} \widehat{\phi}_{2}, \\
& \left(\frac{d}{d t}+v_{i n}\right) \widehat{\phi}_{1}=\frac{k_{y}}{k_{1}} \widehat{n}_{1}+\widehat{\phi}_{0} \widehat{\phi}_{2}, \\
& \left(\frac{d}{d t}+v_{i n}\right) \widehat{\phi}_{2}=-\frac{3}{4} \frac{k_{y} k_{1}}{k_{2}^{2}} \widehat{n}_{2}-\frac{3}{4}\left(\frac{k_{y}}{k_{2}}\right)^{2} \widehat{\phi}_{0} \widehat{\phi}_{1}, \\
& \left(\frac{d}{d t}+4 k_{x}^{2} D\right) \widehat{n}_{0}=\widehat{n}_{1} \widehat{\phi}_{1}, \\
& \left(\frac{d}{d t}+k_{1}^{2} D\right) \widehat{n}_{1}=\frac{k_{y}}{k_{1}} \widehat{\phi}_{1}-\widehat{n}_{0} \widehat{\phi}_{1} \\
& \left.\left(\frac{d}{d t}+k_{2}^{2} D\right) \widehat{n}_{2}=-\frac{3}{4} \frac{k_{1}^{2}}{3 k_{x}^{2}+k_{y}^{2}}\right) \widehat{n}_{2} \widehat{\phi}_{0}, \\
& k_{1}+\widehat{n}_{1} \widehat{\phi}_{0},
\end{aligned}
$$

where

$$
\begin{aligned}
& \widehat{\phi}_{0}=\frac{k_{x} k_{y} \sqrt{3 k_{x}^{2}+k_{y}^{2}}}{\sqrt{3} k_{1}} \phi_{0}, \quad \widehat{\phi}_{1}=\frac{k_{x} k_{y}}{\sqrt{2}} \phi_{1}, \\
& \widehat{\phi}_{2}=\sqrt{\frac{3}{2} \frac{k_{x} k_{y} \sqrt{3 k_{x}^{2}+k_{y}^{2}}}{2 k_{1}} \phi_{2}} \\
& \widehat{n}_{0}=\frac{k_{x} k_{y}}{k_{1}} n_{0}, \quad \widehat{n}_{1}=\frac{k_{x} k_{y}}{\sqrt{2} k_{1}} n_{1}, \\
& \widehat{n}_{2}=\sqrt{\frac{2}{3}} \frac{k_{x} k_{y} \sqrt{3 k_{x}^{2}+k_{y}^{2}}}{k_{1}^{2}} n_{2} .
\end{aligned}
$$

For the simplicity of notation hereafter, we will drop the hat symbol. Now we are interested in finding out the nonlinear evolution of the long wavelength RT mode amplitude in the absence of any dissipation and ion neutral collision, i.e. in the inertial regime. For the analytical progress, we drop the higher density perturbation $n_{2}$; the term 'higher' means perturbation with relatively high $k$, i.e. short wavelength. This assumption does not violate any global behavior of the nonlinear interaction or the conservation property; only the definition of turbulent kinetic energy and the associated dissipation changed slightly. Then, without any loss of generality, 
the self consistent evolution of instability and shear flow becomes:

$$
\begin{aligned}
& \frac{d^{2} \phi_{1}}{d t^{2}}-\alpha_{1}^{2} \phi_{1}+\frac{1}{2} \phi_{1}^{3}=-\left(2 \alpha_{2}+\frac{1}{2}\right) \phi_{0}^{2} \phi_{1} \\
& \frac{d \phi_{0}}{d t}=\sqrt{\alpha_{2}} \phi_{0} \phi_{1}
\end{aligned}
$$

where $\alpha_{1}=k_{y} / k_{1}$ and $\alpha_{2}=3 k_{y}^{2} / 4 k_{2}^{2}$.

From Eqs. (24) and (25) we observed that the fundamental mode amplitude $\phi_{1}$ and shear flow amplitude $\phi_{0}$ are nonlinearly coupled. Furthermore, the mode coupling effect introduces a cubic nonlinearity in the evolution equation. First, in the simplest level, we shall discuss the effect of shear flow on the RT mode analytically. Therefore, in the present discussion, we are not solving a shear flow evolution equation; instead we assume $\phi_{0}$ as a parameter in Eq. (24). However, the evolution of shear flow has been discussed in detailed numerically in the Sect. 7. With the above mentioned assumption, we can solve exactly Eq. (24) analytically. From Eq. (24), it is now clear that in the absence of nonlinearity and shear flow, we recover the linearly unstable RT mode with the usual growth rate in the inertial regime. In the presence of shear flow, we can see that linear RT growth reduces with an increasing amplitude in $\phi_{0}$ and ultimately becomes zero, thereby indicating that linear RT mode stabilizes for a certain value of shear flow. Needless to say, due to the nonlinearity, the stabilization of RT mode is quite different when the nonlinearity and shear flow work against each other and competition between them ultimately saturates the mode. Therefore, in presence of nonlinearity and shear flow, evolution of the system is represented by

$\frac{d^{2} \phi_{1}}{d t^{2}}-\beta^{2} \phi_{1}+\frac{1}{2} \phi_{1}^{3} \approx 0$

where $\beta^{2}=\alpha_{1}^{2}-\left(2 \alpha_{2}+1 / 2\right) \phi_{0}^{2}$. From the definition of $\beta$, it is clear that $\phi_{0}$ has a stabilizing effect on linear RT mode. Solution of the Eq. (26) is given by

$\phi_{1}(t)=2 \beta \operatorname{sech}\left[\beta\left(t-t_{0}\right)\right]$.

Note that $t_{0}$, the time of maximum amplitude, is arbitrary. Since amplitude $\phi_{1}$ of the solution is proportional to $\beta$, we can see that, in reality, as shear flow grows, the amplitude of the nonlinear mode decreases and thereby saturate the growing RT mode in the inertial regime.

Next, we can concentrate on collisional regime $\partial / \partial t \ll$ $v_{i n}$. Assuming $v_{i n} \sim D \sim v$ for simplicity, and taking shear flow $\left(\phi_{0}\right)$ as a parameter as before, we find the approximate evolution equation for the collisional RT mode as

$$
\frac{d \phi_{1}}{d t} \approx \eta \phi_{1}-\zeta \phi_{1}^{3}
$$

where

$$
\eta=\left[\frac{\alpha_{1}^{2}}{2 v}-\frac{v}{2}-\frac{1}{2 v}\left(2 \alpha_{2}+\frac{k_{1}^{2}}{4 k_{x}^{2}}\right) \phi_{0}^{2}\right], \quad \zeta=\frac{k_{1}^{2}}{8 k_{x}^{2} v} .
$$

Note that here we have taken $\phi_{0}$ as a parameter to solve the nonlinear equation, i.e. we have not self consistently solved the shear flow evolution equation and instead, we are assuming shear flow is present in the system due to nonlinear interactions and we took $\phi_{0}$ as a flow parameter. For the different values of $\phi_{0}$, we are investigating its influence on the other modes. We can think of a situation as a 'nonmodal' approach to analyze the shear flow recently used by plasma physicists (Hassam, 1992; Volponi et al., 2000). In this method, one is using a shear flow profile, i.e. $\phi_{0}(x)=v_{\perp 0}^{\prime} x^{2} / 2$ (for simplicity), where $v_{\perp 0}^{\prime}$ is constant, to show how the amplitude of the mode evolves in time, in presence of a given flow (in this particular example, $\left.\boldsymbol{v}_{0}(x)=v_{\perp 0}^{\prime} x \boldsymbol{e}_{y}\right)$. For the present problem we can mockup this situation considering a sinusoidal spatial equilibrium flow profile $\phi_{e q}(x)=\phi_{0} \sin k x$.

Solution of the Eq. (28) is

$\phi_{1}(t)=\sqrt{\eta v} \operatorname{sech}^{1 / 2}\left(2 \eta t+t_{1}\right) \exp \left(\frac{1}{2} \eta t+t_{1}\right)$,

where $t_{1}$ is an arbitrary constant. Note that, in the absence of nonlinearity and shear flow we see (from Eq. 28) that the collisional RT mode is growing exponentially with the usual growth rate $\sim\left(k_{y} / k_{1}\right)^{2}\left(g / L_{n} v\right)$ and this growth suffers a damping due to the presence of a $v / 2$ term in the expression of $\eta$. The origin of the damping term is the electron ion collision effect that we have considered in this case $\left(v_{i n} \sim D \sim v\right)$. However, one must emphasize that for a large scale solution, growth rate of the mode overpowers the damping rate and hence one needs to find out alternative mechanism for the mode saturation. In this calculation we have already incorporated the shear flow (e.g. see the expression of $\eta$ ) which is reducing the RT mode growth rate and therefore, can serve as a candidate of saturation. Here also, we can estimate the critical value of shear flow for which the collisional RT mode is marginally stable. At a first glance it might appear that due to the exponential time behavior in the solution (Eq. 29), the collisional RT mode may not be saturated. A careful inspection of the solution shows that the mode amplitude asymptotically decays in time.

It is very surprising to see the nice symmetric solution (see Eq. 27) for the nonlinear equation. This symmetry may be destroyed if we include a large number of modes in the calculations. Mode coupling with higher harmonics will extract energy from the mode, causing irreversibility, and also a dissipativing effect, such as viscosity and diffusion, can break this symmetry. Therefore, there is a possibility that the mode coupling to higher harmonics can enhance the role of dissipation. One can show that energy moves from the two modes $k_{1}, k_{2}$ to the shear mode and the flattening term, and then returns again during evolution when the shear is small; yet anything that disrupts this exchange of energy will destroy this reversibility and yield transport. Therefore, these effects are important for the estimation of transport coefficients. 


\section{Shear-flow stabilization with higher harmonics}

In the previous section we have assumed that in the higher harmonics of density perturbations, $n_{2}$ was absent. In the present analysis we consider finite $n_{2}$, but assume that the damping rate of the $\left(\phi_{2}, n_{2}\right)$ mode is much higher than the characteristic time of evolution of the whole system. In the present model we have taken a linear damping mechanism as a perpendicular diffusion in the continuity equation and ion-neutral collisions $\left(v_{i n}\right)$ in the vorticity equation. We denote $4 k_{x}^{2} D=D_{0}, k_{1}^{2} D=D_{1}, k_{2}^{2} D=D_{2}$ and since $k_{2}>$ $k_{1} \gg 2 k_{x}, D_{2}$ is larger. Therefore, the modes with dissipation coefficient $D_{2}$ are heavily damped mode. In principle, the viscosity effect should also be present as a linear damping mechanism and similar to diffusion, it is very effective to dissipate the high $k$ modes. Keeping this effect in mind, we propose that in our model, $v_{i n}$ will represent $v_{0}, v_{1}, v_{2}$ as the dissipation rates for the three harmonics in the vorticity equations. Assuming $D_{2}, v_{2} \gg \alpha_{1}$, indicates that the damping rate for higher harmonics is higher than the growth rate. Consequently, we can write Eqs. (20) and (23) as:

$\nu_{2} \phi_{2}+\frac{3}{4} \alpha_{1} \bar{\alpha}_{2} n_{2}=-\frac{3}{4} \bar{\alpha}_{2} \phi_{0} \phi_{1}$,

$D_{2} n_{2}+\frac{4}{3} \alpha_{1} \phi_{2}=\phi_{0} n_{1}$

from which we obtain

$\phi_{2}=-\frac{\left(D_{2} \alpha_{2} \phi_{1}+\alpha_{1} \alpha_{2} n_{1}\right) \phi_{0}}{\nu_{2} D_{2}-\alpha_{1}^{2} \bar{\alpha}_{2}}$,

$n_{2}=\frac{\left(v_{2} n_{1}+\alpha_{1} \bar{\alpha}_{2} \phi_{1}\right) \phi_{0}}{v_{2} D_{2}-\alpha_{1}^{2} \bar{\alpha}_{2}}$,

where $\alpha_{2}=3 \bar{\alpha}_{2} / 4$. Substituting $\phi_{2}$ and $n_{2}$ into the dynamical equation for $n_{1}$ and $\phi_{1}$ (Eqs. 22 and 19) we have

$\frac{d n_{1}}{d t}=-K_{1} n_{1}+K_{2} \phi_{1}$
$\frac{d \phi_{1}}{d t}=-K_{3} \phi_{1}+K_{4} n_{1}$,

where

$$
\begin{aligned}
& K_{1}=D_{1}+\left(\frac{v_{2} \alpha_{3} \phi_{0}^{2}}{v_{2} D_{2}-\alpha_{1}^{2} \bar{\alpha}_{2}}\right), \\
& K_{2}=\alpha_{1}-n_{0}-\left(\frac{\alpha_{1} \bar{\alpha}_{2} \alpha_{3} \phi_{0}^{2}}{v_{2} D_{2}-\alpha_{1}^{2} \bar{\alpha}_{2}}\right), \\
& K_{3}=v_{1}+\left(\frac{D_{2} \alpha_{2} \phi_{0}^{2}}{v_{2} D_{2}-\alpha_{1}^{2} \bar{\alpha}_{2}}\right), \\
& K_{4}=\alpha_{1}-\left(\frac{\alpha_{1} \alpha_{2} \phi_{0}^{2}}{v_{2} D_{2}-\alpha_{1}^{2} \bar{\alpha}_{2}}\right),
\end{aligned}
$$

and $\alpha_{3}=3 k_{1}^{2} / 4\left(3 k_{x}^{2}+k_{y}^{2}\right)$. We have assumed that $\nu_{2} \sim$ $D_{2}>\alpha_{1}$ and we can easily find $\bar{\alpha}_{2}=\left(k_{y} / k_{2}\right)^{2}<1$; therefore, $\left(v_{2} D_{2}-\alpha_{1}^{2} \bar{\alpha}_{2}\right)$ is always positive. It is now clear from the definition of $K$ that the growth of $\phi_{0}$ will increase the damping coefficients and simultaneously reduce the destabilizing terms $K_{2}$ and $K_{4}$, leading to the saturation of the instability and increasing the characteristic time of evolution.

\section{Secondary instability}

It is mentioned in the introduction that there exists a wellknown and successful example of a low dimensional model namely, the Lorenz set for Benard convection in unstable stratified fluid. In the Lorenz model (Lorenz, 1963), the only flow structure taken into account is a regular chain of vortices, $\phi=\phi_{1} \sin k_{x} \sin k_{y} y$. As a density structure, it includes both profile flattening (the amplitude $n_{0}$ ) and a spike, such as convective deformation (the amplitude $n_{1}$ ), so that $n=n_{0} \sin 2 k_{x} x+n_{1} \sin k_{x} x \cos k_{y} y$. Therefore, the triple $\left(n_{0}, n_{1}, \phi_{1}\right)$ constitute a complete Lorenz set. A similar model has been used before in the ionospheric turbulence to study the low dimensional chaos (Huba et al., 1986). In the present 6 ODE problem, we assume that for certain conditions the system evolves to this Lorenz attractor branches for any initial amplitude $\phi_{0}, n_{2}, \phi_{2}$ that decays to zero. Now, our aim is to study the stability of the fixed point given by $\phi_{0}=n_{2}=\phi_{2}=0$ and $n_{0}, n_{1}, \phi_{1}$ in Eqs. (12)-(17) to small amplitude perturbations of three variables $\phi_{0}, n_{2}, \phi_{2}$.

First, we shall show that in the truncated Fourier mode representation, the fundamental mode $\left(\phi_{1}, n_{1}\right)$ is linearly unstable. A linear version of Eqs. (13) and (16) reads

$$
\begin{aligned}
& k_{1}^{2} \frac{d \phi_{1}}{d t}=k_{y} n_{1}-v_{i n} k_{1}^{2} \phi_{1} \\
& \frac{d n_{1}}{d t}=k_{y} \phi_{1}-k_{1}^{2} D n_{1} .
\end{aligned}
$$

Taking perturbations in the form of $\phi_{1} \sim n_{1} \sim \exp \left(\gamma_{p} t\right)$, where $\gamma_{p}$ is the growth rate for the primary RT mode, the linear dispersion relation may be obtained as

$\gamma_{p}^{2}+\left(v_{i n}+k_{1}^{2} D\right) \gamma_{p}-\frac{k_{y}^{2}}{k_{1}^{2}}+k_{1}^{2} v_{i n} D=0$

From the dispersion relation, it is clear that the condition for the first instability is

$\frac{k_{y}^{2}}{k_{1}^{2}}>k_{1}^{2} v_{i n} D \quad$ or $\quad \frac{k_{1}^{4} v_{i n} D}{k_{y}^{2}} \leq 1, \quad$ or $\quad Q \geq 1$

where the equal sign is for the onset of the instability and $Q=k_{y}^{2} / k_{1}^{4} v_{\text {in }} D$.

Next, let us find the quasi steady state of $\left(\phi_{1}, n_{1}, n_{0}\right)$ for a Lorenz set of equations which nonlinearly saturates to form a convective cell or bubble-like solution. Nonlinear equations of this steady state is obtained by putting $d / d t \approx 0$. Using Eqs. (13), (16) and (15), we have

$$
\begin{aligned}
& \frac{k_{y}}{k_{1}^{2}} n_{1}-v_{i n} \phi_{1}=0, \\
& k_{y} \phi_{1}-k_{x} k_{y} \phi_{1} n_{0}-k_{1}^{2} D n_{1}=0, \\
& \frac{1}{2} k_{x} k_{y} n_{1} \phi_{1}-4 k_{x}^{2} D n_{0}=0 .
\end{aligned}
$$


The solutions of these nonlinear equations provide the equilibrium around which we will perturbed the system. Now, for clarity, we denote these solutions $n_{0}, \phi_{1}, n_{1}$ as $n_{00}, \phi_{10}, n_{10}$, so that the solutions become

$n_{00}=\frac{1}{k_{x}}\left(1-\frac{1}{Q}\right)$

$\phi_{10}= \pm \sqrt{\frac{8 D}{v_{i n} k_{1}^{2}}\left(1-\frac{1}{Q}\right)}$,

$n_{10}= \pm \frac{v_{i n} k_{1}^{2}}{k_{y}} \sqrt{\frac{8 D}{v_{i n} k_{1}^{2}}\left(1-\frac{1}{Q}\right)}$,

where the upper and lower sign indicates the handedness or the sense of rotation of the cells or bubbles.

From the solutions given in Eqs. (42)-(44), we can see that for a weak ion-neutral collision effect, the flow velocity term $\phi_{10}$ becomes large so that the $\boldsymbol{E} \times \boldsymbol{B}$ rotation rate in the bubble can exceed the linear growth rate, i.e. $k_{x} k_{y} \phi_{10}>\gamma_{p}$. Also the gradient in the $y$ direction of the density becomes very strong with $k_{y} n_{10}>1$. Thus, we may expect high flow velocity and steep density gradient in the fundamental mode $\left(n_{1}, \phi_{1}\right)$ to become unstable to a 'secondary instability'. We assume the secondary perturbations are of the form $\left(\phi_{0}, \phi_{2}, n_{2}\right) \exp \left(\gamma_{s} t\right)$, where $\gamma_{s}$ is the growth rate for the secondary mode. Therefore, the dynamical evolution of these modes may be obtained from linearized Eqs. (12), (14) and (17) and are given by

$\gamma_{s} \phi_{0}=-\frac{3}{4} k_{x} k_{y} \phi_{10} \phi_{2}-v_{i n} \phi_{0}$,

$\gamma_{s} \phi_{2}=-\frac{k_{y}}{k_{2}^{2}} n_{2}-\frac{k_{x} k_{y}^{3}}{2 k_{2}^{2}} \phi_{10} \phi_{0}-v_{i n} \phi_{2}$,

$\gamma_{s} n_{2}=-k_{y} \phi_{2}+\frac{1}{2} k_{x} k_{y} n_{10} \phi_{0}-k_{2}^{2} D n_{2}$.

The dispersion relation for the secondary mode is the cubic equation for $\gamma_{s}$ and is given by

$\gamma_{s}^{3}+\beta_{1} \gamma_{s}^{2}+\beta_{2} \gamma_{s}+\beta_{3}=0$,

where

$$
\begin{aligned}
\beta_{1}= & 2 v_{i n}+k_{2}^{2} D, \\
\beta_{2}= & v_{i n}\left(2 D k_{2}^{2}+v_{i n}\right)-\frac{k_{y}^{2}}{k_{2}^{2}}-3 \frac{D}{v_{i n}} \frac{k_{x}^{2} k_{y}^{4}}{k_{1}^{2} k_{2}^{2}}\left(1-\frac{1}{Q}\right), \\
\beta_{3}= & v_{i n}^{2} D k_{2}^{2}-\frac{k_{y}^{2}}{k_{2}^{2}} v_{i n} \\
& -3\left[\frac{k_{y}^{2} k_{x}^{2}}{k_{2}^{2}} D+\frac{D^{2}}{v_{i n}} \frac{k_{x}^{2} k_{y}^{4}}{k_{1}^{2}}\right]\left(1-\frac{1}{Q}\right) .
\end{aligned}
$$

In the above expressions, $(1-1 / Q)$ determines the strength of the primary flow $\phi_{10}$ and gradients of $n_{10}, n_{00}$, and, therefore, the stability of the steady state in Eqs. (42)-(44). The cubic Eq. (48) is unstable for $\beta_{3}<0$ and one unstable root may be $\gamma_{s 1} \sim-\beta_{3} / \beta_{2}$, and the solution evolves to a

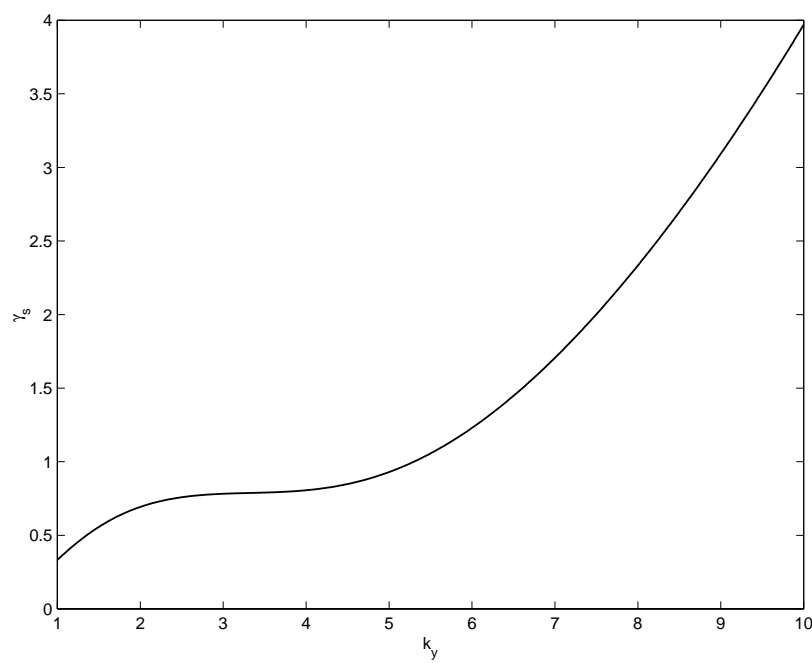

Fig. 1. growth rate for the secondary instability against $k_{y}$, for $k_{x}=$ $1.0, v_{i n}=0.3, D=0.2$, which indicates that secondary mode grows in the short scale regime.

new state with finite steady shear flow. In this case, a stability boundary can be determined by setting $\beta_{3}=0$. For $\beta_{3}>\beta_{1} \beta_{2}$, there is a bifurcation with a pair of complex conjugate roots becoming unstable for $\operatorname{Re} \gamma_{s}>0$. Figure 1 shows the growth rate for the secondary instability $\gamma_{s}$ against $k_{y}$ for the typical values of $v_{i n}=0.3$ and $D=0.2$ and $k_{x}=1.0$.

\section{Collisional nonlinearity in shear flow generation}

In the previous analysis of shear flow generation, we have found that the consideration of the mode coupling term shows that the $\boldsymbol{E} \times \boldsymbol{B}$ convection of vorticity (which essentially comes from the ion polarization drift nonlinearity) generates the convective flows $\phi_{0} \sin \left(k_{x} x\right)$. At the same time, we have ignored the contribution of the Pedersen drift nonlinearity $(\nabla n \cdot \nabla \phi$, which arises due to ion-neutral collision effect) in the flow generation. These two nonlinearities are different in their character, for example $\boldsymbol{e}_{z} \times \nabla \phi \cdot \nabla \nabla_{\perp}^{2} \phi$, is normally known as a vector nonlinearity and $\nabla n \cdot \nabla \phi$ is a scalar nonlinearity; therefore, they contribute differently in the flow generation. For the two modes we have considered, we have seen that $\boldsymbol{e}_{z} \times \nabla \phi \cdot \nabla \nabla_{\perp}^{2} \phi$ generates an anti-symmetric flow in the flow profile and similarly, we can see that $\nabla n \cdot \nabla \phi$ generates a symmetric flow profile $\sim \cos \left(k_{x} x\right)$. Therefore, to find out the role of both of the nonlinearities in the flow generation, we have to incorporate symmetric as well as anti-symmetric flows with different amplitudes. In such a scenario, instead of 6 ODE, we have to solve 7 ODE system for the amplitude evolution:

$$
\begin{aligned}
& \frac{d \widehat{\phi}_{0}}{d t}=-\widehat{\phi}_{1} \widehat{\phi}_{2}-\frac{v_{i n} k_{x} k_{1}}{k_{y}} \widehat{n}_{0} \widehat{\bar{\phi}}_{0}-v_{i n} \widehat{\phi}_{0}, \\
& \frac{d \widehat{\phi}_{1}}{d t}=\frac{k_{y}}{k_{1}} \widehat{n}_{1}+\widehat{\phi}_{0} \widehat{\phi}_{2}-\frac{3}{2} \frac{v_{i n} k_{1} k_{x}}{k_{y}\left(3 k_{x}^{2}+k_{y}^{2}\right)} \widehat{n}_{2} \widehat{\bar{\phi}}_{0}-v_{i n} \widehat{\phi}_{0},
\end{aligned}
$$




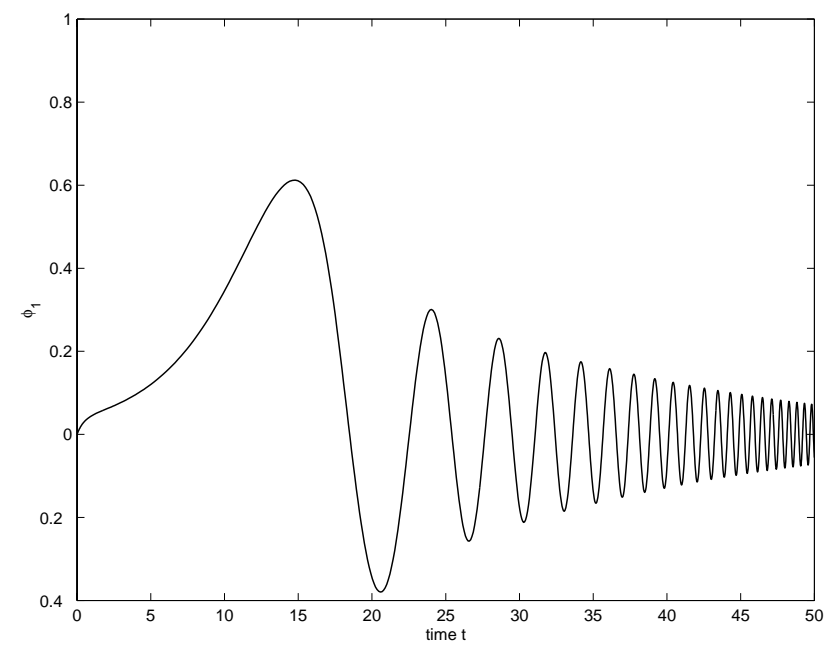

Fig. 2. Potential fluctuation amplitude $\phi_{1}$, of the fundamental mode for $k_{x}=0.4, k_{y}=1.4, v_{i n}=0.24$ and $D=0.2$, which indicates that long scale radial RT mode saturates in time.

$\frac{d \widehat{\phi}_{2}}{d t}=-\frac{3}{4} \frac{k_{y} k_{1}}{k_{2}^{2}} \widehat{n}_{2}-\frac{3}{4}\left(\frac{k_{y}}{k_{2}}\right)^{2} \widehat{\phi}_{0} \widehat{\phi}_{1}-v_{i n} \widehat{\phi}_{2}$,

$\frac{d \widehat{n}_{0}}{d t}=\widehat{n}_{1} \widehat{\phi}_{1}-4 k_{x}^{2} D \widehat{n}_{0}$,

$\frac{d \widehat{n}_{1}}{d t}=\frac{k_{y}}{k_{1}} \widehat{\phi}_{1}-\widehat{n}_{0} \widehat{\phi}_{1}-\frac{3}{4}\left(\frac{k_{1}^{2}}{3 k_{x}^{2}+k_{y}^{2}}\right) \widehat{n}_{2} \widehat{\phi}_{0}-k_{1}^{2} D \widehat{n}_{1}$,

$\frac{d \widehat{n}_{2}}{d t}=-\frac{4}{3} \frac{k_{y}}{k_{1}} \widehat{\phi}_{2}+\widehat{n}_{1} \widehat{\phi}_{0}-k_{2}^{2} D \widehat{n}_{2}$,

$\frac{d \widehat{\bar{\phi}}_{0}}{d t}=\frac{v_{i n} k_{x} k_{1}}{k_{y}}\left(\widehat{\phi}_{0} \widehat{n}_{0}+\frac{2}{3} \widehat{n}_{1} \widehat{\phi}_{2}+\frac{1}{2} \widehat{n}_{2} \widehat{\phi}_{1}\right)-v_{i n} \widehat{\bar{\phi}}_{0}$,

where $\bar{\phi}_{0}$ has the same scaling as $\phi_{0}$. Note that the amplitude evolution equation for $\bar{\phi}_{0}$ (Eq. 55) arises because we have added the symmetric flow term $\bar{\phi}_{0} \cos \left(k_{x} x\right)$ in our potential representation.

We must mention that due to the presence of collisional nonlinearity mentioned above, the system no longer supports invariants; therefore, it is practically impossible to analyze 7 ODE system analytically. We have solved Eqs. (49)-(55) numerically using a standard ODE solver routine in MATLAB. The initial conditions are taken as $n_{1}, \phi_{0}, \bar{\phi}_{0}=0.1$ and $n_{0}, n_{2}, \phi_{1}, \phi_{2}=0$. The nonzero initial values $\phi_{0}, \bar{\phi}_{0}$ implies that we have initialized the system with a seed shear flow to study their evolutions. The results are shown in the Figs. 2-4 for typical parameters.

We have seen from Eq. (55) that the symmetric shear flow is solely driven by collisional nonlinearity; therefore, in absence of ion-neutral collision, no spatially symmetric shear flow will develop $\left(v_{i n}=0, \bar{\phi}_{0}=0\right)$. For large $v_{i n}(\sim 1)$, one might expect that symmetric shear flow will be large, but numerical results show that for large $v_{i n}$, both symmetric and anti-symmetric flow decays. Even for moderate values of $v_{\text {in }}(\sim 0.5)$ numerical result shows the symmetric shear flow amplitude is lower than antisymmetric flow amplitude.

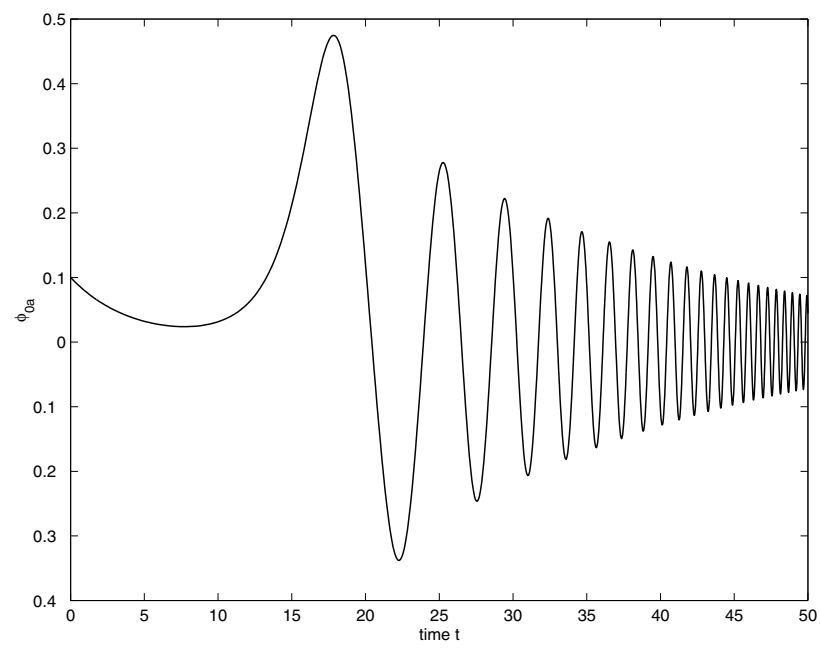

Fig. 3. Temporal evolution of the anti-symmetric shear flow amplitude for $k_{x}=0.4, k_{y}=1.4, v_{i n}=0.24$ and $D=0.2$, which indicates that the initial seed flow has transient growth and then oscillatory decay due to finite dissipation present in the system.

Perhaps for a higher $v_{i n}$ and finite $D, n \phi$ nonlinearity for various modes decays faster than the $\phi_{1} \phi_{2}$ nonlinearity; therefore, the collisional driving term for the flow is weaker than the convective driving term. Thus, even in the collisional regime, the Pedersen nonlinearity may not play a significant role in the shear flow generation and one can justify the neglecting of the contribution from the symmetric flow in the analytical calculation in Sect. 4.

\section{Discussion and conclusion}

Perhaps the most important conclusion of this work is that the RT mode nonlinearly saturates in both a collisionless and collisional regime by generating shear flow which has been demonstrated analytically. First, we have started with a well documented linear instability dispersion relation from which collisionless and collisional RT growth rate had been derived. Next, we have taken a model for few mode calculations which represent the essential nonlinear physics of the RT mode e.g. $\boldsymbol{E} \times \boldsymbol{B}$ convection nonlinearly generates the shear flow. At this point we must emphasized that the finite mode calculation of shear flow has the deficiency that it ignores the contribution of the higher modes to the generation of $\phi_{0}$. Thus, it would appear that during the evolution when the higher modes also have significant amplitudes, the inference regarding the magnitude of shear flow may be quantitatively incorrect and the whole process of shear flow generation and saturation can, at best, be viewed only qualitatively. However this is not the case. There are many simulation results existing in the literature which show the plots of $\left|\phi_{0}\right|^{2}$ against time from the complete numerical simulation and few mode calculations (Das et al., 1997). The results show reasonably good quantitative agreement, which can be understood on the basis of Kolmogorov's cascade mechanisms of 


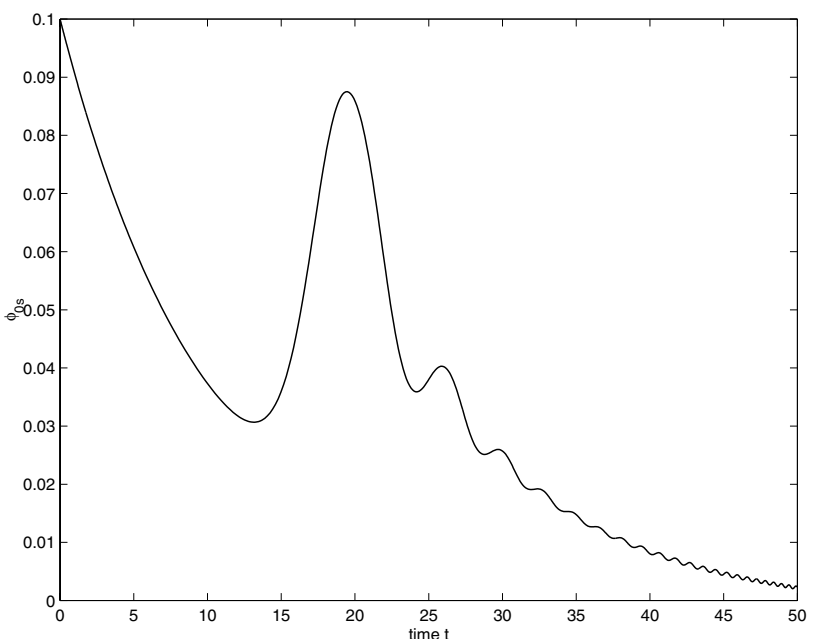

Fig. 4. Temporal evolution of the symmetric shear flow amplitude for $k_{x}=0.4, k_{y}=1.4, v_{i n}=0.24$ and $D=0.2$, which indicates that the initial seed flow does not have significant growth.

the power transfer. The dominant transfer takes place only through the interaction between $\phi_{1}$ and $\phi_{2}$. The second and higher order effects or other modes influencing the amplitude of $\phi_{1}$ and $\phi_{2}$ and thereby contributing to the magnitude of shear flow generated is also negligible, as the dominant change in the amplitude of $\phi_{1}$ comes through the linear growth of the RT mode.

We have done a model calculation to show that due to free energy generated in the quasi-steady bubbles, they are unstable to secondary instabilities. The mode coupling equations contain a Lorenz attractor on a sub-manifold. Taking the Lorenz branch as a fixed point, we find the dynamical evolution of the remaining three variables in the 6-D phase space. We have shown that nonlinearly formed convective cells or bubbles are unstable to secondary instabilities due to the strong gradient developed by them which trends to fragment them into short scales (smaller than the convective cell size). The resulting size of the short scale structure is not calculated, since it involves the initial amplitude and nonlinear development of the secondary instabilities. One of the basic mechanism of the onset of turbulence is the destruction of coherent structures and it may be that the secondary instability mechanism is one of such processes which destroys the structures.

We have not addressed the question of total transport of energy in this study. Most of our discussion, in fact, is involved with a model calculation to illustrate the basic physical fact. To pin down these facts more quantitatively, more investigation is needed, for example for the secondary instability, one must consider a 2-D bubble structure as an equilibrium and then perturb it with short scale perturbations in more systematic way. Such a calculation might give us the saturation length scale of such an instability and help us to calculate the transport coefficients. However, from the model calculation, we expect that only moderate scale bubbles can survive, since large-scale bubbles are ruled out due to secondary instability. All of these conclusions are qualitative. In the future, we hope to address this problem more quantitatively by numerical simulation to clarify some of these issues.

Acknowledgements. Thanks are due to B. P. Pandey and A. K. Sinha for some helpful discussions.

\section{References}

Amatucci, W. E., Walker, D. N., Ganguli, G., et al., Plasma response to strongly sheared flow, Phys. Rev. Lett., 77, 1978, 1996; and reference therein.

Arons, J. and Lea, S. M., Accretion on to magnetized neutron stars: structure and interchange instability of a model magnetosphere, Astrophys. J., 207, 914, 1976.

Basu, B., Generalized Rayleigh-Taylor instability in the presence of time dependent equilibrium, J. Geophys. Res., 102, 17305, 1997.

Beyer, P. and Spatschek, K. H., Center manifold theory for the dynamics of L-H transition, Phys. Plasmas, 3, 995, 1996.

Burlaga, L. F., Intermittent turbulence in the solar wind, J. Geophys. Res., 96, 5847, 1991.

Chaturvedi, P. K. and Ossakow, S. L., Non-linear theory of the collisional Rayleigh-Taylor instability in equatorial spread F ,Geophys. Res. Lett., 4, 558, 1977.

Das, A., et al., Nonlinear saturation of Rayleigh-Taylor instability, Phys. Plasmas, 4, 1018, 1997.

Finn, J. M., Nonlinear interaction of Rayleigh-Taylor and shear instabilities, Phys. Fluids B, 5, 415, 1993.

Finn, J. M., Drake, J. F., and Guzdar, P. N., Instability of fluid vortices and generation of shear flow, Phys. Fluids B, 4, 2758, 1992.

Flaherty, J. P., Seyler, C. E., and Trefethen, L. N., Large amplitude transient growth in the linear evolution of equatorial spread $\mathrm{F}$ with a sheared zonal flow, J. Geophys. Res., 104, 6843, 1999.

Guzdar, P. N., Satyanarayana, P., Huba, J. D., and Ossakow, S. L., Influence of velocity shear on Rayleigh-Taylor instability, Geophys. Res. Letts., 9, 547, 1982.

Hassam, A. B., Hall, W., Huba, J. D., and Keskinen, M. J., Spectral characteristics of interchange turbulence, J. Geophys. Res., 91, 13513, 1986

Hassam, A. B., Nonlinear stabilization of the Rayleigh-Taylor instability by external velocity shear, Physics Fluids, 4, 485, 1992.

Hermiz, K. B., Guzdar, P. N., and Finn, J. M., Improved low order model for shear flow driven by Rayleigh-Benard convection, Phys. Rev. E, 51, 325, 1995.

Horton, W., Hu, G., and Laval, G., Trubulent transport in mix states of convective cells and shear flows, Phys. Plasmas, 3, 2912, 1996.

Howard, L. N. and Krshanamurti, R., large scale-flow in turbulent convection: a mathematical model, J. Fluid Mech., 170, 385, 1986.

Huba, J. D., Hassam, A. B., Schwartz, I. B., and Keskinen, M. J., Ionospheric turbulence: Interchange instabilities and chaotic fluid behavior, Geophys. Res. Lett.,12, 65, 1985.

Huba, J. D., Bernhardt, P. A., Ossakaw, S. L., and Zaselak, S. T. The Rayleigh-Taylor instability is not damped by recombination in the F region, J. Geophys. Res., 101, 24553, 1996.

Huba, J. D, Finite larmor radius magnetohydrodynamics of the Rayleigh-Taylor instability, Phys. Plasmas, 3, 2523, 1996.

Keskinen, M. J., Sudan, R. N., and Ferch, R. L., Temporal and spatial power spectrum studies of numerical simulations of type II 
gradient drift irregularities in the equatorial electrojet, J. Geophys. Res., 84, 1419, 1979.

Lorenz, E. N., Deterministic nonperiodic flow, J. Atmos. Sci., 20, 130, 1963.

Penano, J. R., Ganguli, G., Amatucci, W. E., et al., Velocity shear driven instabilities in a rotating plasma layer, Phys. Plasmas, 5, 4377, 1998.

Pogutse, O., et al., The resistive interchange convection in the edge of tokamak plasma, Plasma Phys. Control Fusion, 36, 1963, 1994.

Satyanarayana, P., Lee, Y. C., and Huba, J. D., Phys. Fluids, 30, 81, 1987.

Sazonov, S. V., Dissipative structures in the F-region of the equa- torial ionsphere generated by Rayleigh-Taylor instability, Planet. Space Sci., 39, 1667, 1991.

Sudan, R. N. and Keskinen, M. J., Unified theory of power spectrum of intermidiate wavelength ionospheric electron density fluctuations, J. Geophys. Res., 89, 9840, 1984.

Volponi, F., Yoshida, Z., and Tatsuno, T., Shear flow induced stabilization of kinklike modes, Phys. Plasmas, 7, 2314, 2000.

Wilcock, W. S. D. and Whitehead, J. A., The Rayleigh-Taylor instability of an embedded layer of low viscosity fluid, J. Geophys. Res., 96, 12193, 1991.

Zalesak, S. T., Ossakow, S. L., and Chturvedi, P. K., Nonlinear equatorial spread F: The effect of neutral winds and background Pedersen conductivity, J. Geophys. Res. Lett., 87, 151, 1982. 